\title{
On Para-Complex Affine Hyperspheres
}

\author{
Zuzanna Szancer
}

\begin{abstract}
In this paper we introduce a notion of a para-complex affine hypersphere. We give a complete local classification of such hypersurfaces and give several examples. It turns out that every para-complex affine hypersphere can be constructed from (real) affine hyperspheres. As an application, we classify all 2-dimensional para-complex affine hyperspheres.
\end{abstract}

Mathematics Subject Classification. 53A15, 53D15.

Keywords. Affine hypersphere, para-complex structure, para-complex hypersphere.

\section{Introduction}

The main motivation for this paper are results obtained by Dillen et al. [1]. In that paper the authors introduce a notion of a complex affine hypersurface and, in particular, a notion of a complex affine hypersphere. Now, it seems to be natural to ask what happens in a para-complex case. Para-complex structures are widely studied by many authors (see e.g. [2-4]). A concept of a para-complex affine immersion as well as a para-complex affine hypersurface was introduced by Schäfer and Lawn [5].

In this paper we introduce a notion of a para-complex affine hypersphere and give a complete local classification of such hypersurfaces. More precisely, we show that every para-complex affine hypersphere can be locally obtained from two real affine hyperspheres. In particular, we can construct several examples of para-complex affine hyperspheres using well know examples of real affine hyperspheres. As an application we provide examples of 1-dimensional

This Research was financed by the Ministry of Science and Higher Education of the Republic of Poland. 
(in a para-complex sense) para-complex affine spheres and show that they are the only 1-dimensional para-complex affine spheres up to a para-complex equiaffine transformation.

In Sect. 2 we briefly recall basic formulas of affine differential geometry and recall the notion of an affine hypersphere. Since para-complex affine hypersufaces are hypersurfaces of a real codimension two, we recall also a concept of an affine hypersurface of codimension two.

In the first part of Sect. 3 we recall some basic concepts related to paracomplex geometry (for details we refer to [5-7]). Later, using similar methods like in [1] we introduce a notion of affine normal fields for para-complex affine hypersurfaces and study several basic properties of hypersurfaces equipped with such vector field.

The Sect. 4 contains main results of this paper. In this section we introduce a notion of a para-complex affine hypersphere and prove classification theorems. Especially, we shall show that there is a strict correspondence between real and para-complex affine hyperspheres. We also give several examples.

\section{Preliminaries}

We briefly recall the basic formulas for affine differential geometry. For more details, we refer to [8]. Let $f: M \rightarrow \mathbb{R}^{n+1}$ be an orientable connected differentiable $n$-dimensional hypersurface immersed in affine space $\mathbb{R}^{n+1}$ equipped with its usual flat connection $\mathrm{D}$. Then for any transversal vector field $C$ we have

$$
\mathrm{D}_{X} f_{*} Y=f_{*}\left(\nabla_{X} Y\right)+h(X, Y) C
$$

and

$$
\mathrm{D}_{X} C=-f_{*}(S X)+\tau(X) C,
$$

where $X, Y$ are tangent vector fields. For any transversal vector field $\nabla$ is a torsion-free connection, $h$ is a symmetric bilinear form on $M$, called the second fundamental form, $S$ is a tensor of type $(1,1)$, called the shape operator and $\tau$ is a 1 -form.

In this paper we assume that $h$ is nondegenerate so that $h$ defines a pseudo-Riemannian metric on $M$. If $h$ is nondegenerate, then we say that the hypersurface or the hypersurface immersion is nondegenerate. We have the following

Theorem 2.1. ([8], Fundamental equations) For an arbitrary transversal vector field $C$ the induced connection $\nabla$, the second fundamental form $h$, the shape operator $S$, and the 1-form $\tau$ satisfy the following equations:

$$
\begin{aligned}
& R(X, Y) Z=h(Y, Z) S X-h(X, Z) S Y, \\
& \left(\nabla_{X} h\right)(Y, Z)+\tau(X) h(Y, Z)=\left(\nabla_{Y} h\right)(X, Z)+\tau(Y) h(X, Z), \\
& \left(\nabla_{X} S\right)(Y)-\tau(X) S Y=\left(\nabla_{Y} S\right)(X)-\tau(Y) S X,
\end{aligned}
$$




$$
h(X, S Y)-h(S X, Y)=2 d \tau(X, Y) .
$$

The Eqs. (2.1), (2.2), (2.3), and (2.4) are called the equation of Gauss, Codazzi for $h$, Codazzi for $S$ and Ricci, respectively.

For an affine hypersurface the cubic form $Q$ is defined by the formula

$$
Q(X, Y, Z)=\left(\nabla_{X} h\right)(Y, Z)+\tau(X) h(Y, Z) .
$$

It follows from the equation of Codazzi (2.2) that $Q$ is symmetric in all three variables.

For a hypersurface immersion $f: M \rightarrow \mathbb{R}^{n+1}$ a transversal vector field $C$ is said to be equiaffine (resp. locally equiaffine) if $\tau=0$ (resp. $d \tau=0$ ). For an affine hypersurface $f: M \rightarrow \mathbb{R}^{n+1}$ with a transversal vector field $C$ we consider the following volume element on $M$ :

$$
\theta\left(X_{1}, \ldots, X_{n}\right)=\operatorname{det}\left[f_{*} X_{1}, \ldots, f_{*} X_{n}, C\right]
$$

for all $X_{1}, \ldots, X_{n} \in \mathcal{X}(M)$. We call $\theta$ the induced volume element on $M$.

When $f$ is nondegenerate, there exists a canonical transversal vector field $C$, called the affine normal (or the Blaschke field). The affine normal is uniquely determined up to sign by the following conditions:

$$
\begin{aligned}
\tau & =0 \quad \text { (i.e. } \mathrm{C} \text { is equiaffine), } \\
\omega_{h} & =\theta,
\end{aligned}
$$

where $\omega_{h}$ is defined by $\omega_{h}\left(X_{1}, \ldots, X_{n}\right)=\left|\operatorname{det}\left[h\left(X_{i}, X_{j}\right)\right]\right|^{1 / 2}$, where $X_{1}, \ldots, X_{n}$ is positively oriented basis relative to the induced volume form $\theta$. The affine immersion $f$ with a Blaschke field $C$ is called a Blaschke hypersurface.

A Blaschke hypersurface $M$ is called an improper affine hypersphere if $S=0$. If $S=\lambda$ id, where $\lambda$ is a nonzero constant, then $M$ is called a proper affine hypersphere.

Remark 2.1. Sometimes it is convenient to weak the condition $\omega_{h}=\theta$ and replace it with $\omega_{h}=c \cdot \theta$, where $c \in \mathbb{R} \backslash\{0\}$. When for some equiaffine vector field $\xi$ we have $\omega_{h}=c \cdot \theta$ then $\xi$ is proportional to the Blaschke field. Namely we have that $\xi^{\prime}:= \pm|c|^{\frac{2}{n+2}} \cdot \xi$ is the Blaschke field. Note also that if the shape operator is proportional to identity then $f$ (with $\xi^{\prime}$ ) is an affine hypersphere. We will often make use of this observation later in this paper.

Let $(M, \nabla)$ and $(\widetilde{M}, \widetilde{\nabla})$ be two differential manifolds of dimension $n$ and $n+p$ with torsion-free affine connections $\nabla$ and $\widetilde{\nabla}$ respectively.

An immersion $f: M \rightarrow \widetilde{M}$ is called an affine immersion if there exists around each point of $M$, a field $\mathcal{N}$ of transversal subspaces of dimension $p$, denoted by $x \mapsto N_{x} \subset T_{f(x)}(\widetilde{M})$ and such that

$$
T_{f(x)}(\widetilde{M})=f_{*}\left(T_{x} M\right)+N_{x}
$$

holds and, for all vector fields $X$ and $Y$ on $M$, we have a decomposition

$$
\widetilde{\nabla}_{X} f_{*} Y=f_{*} \nabla_{X} Y+\alpha(X, Y)
$$


where $\nabla_{X} Y \in T_{x} M$ and $\alpha(X, Y) \in N_{x}$ at each point $x$. We call $N_{x}$ the transversal space and $\alpha$ the affine fundamental form. If $\xi$ is a vector field with values in $\mathcal{N}, \xi_{x} \in N_{x}$, then we write

$$
\widetilde{\nabla}_{X} \xi=-f_{*} S_{\xi} X+\nabla_{X}^{\perp} \xi
$$

where $S_{\xi} X \in T_{x} M$ and $\nabla_{X}^{\frac{1}{X}} \xi \in N_{x}$ at each point $x$. We call $S_{\xi}$ the shape operator for $\xi$, and $\nabla^{\perp}$ the normal connection.

Now, let $\widetilde{M}=\mathbb{R}^{n+2}$ and $\widetilde{\nabla}=D$ be the ordinary flat connection on $\mathbb{R}^{n+2}$. Let $f: M \rightarrow \mathbb{R}^{n+2}$ be an immersion, and $\mathcal{N}: M \ni x \mapsto N_{x}$ be a transversal bundle for the immersion $f$. Immersion $f$ together with the transversal bundle $\mathcal{N}$ we call an affine hypersurface of codimension two. For any local basis $\left\{\xi_{1}, \xi_{2}\right\}$ of $\mathcal{N}$, we can write

$$
\begin{aligned}
D_{X} f_{*} Y & =f_{*}\left(\nabla_{X} Y\right)+h_{1}(X, Y) \xi_{1}+h_{2}(X, Y) \xi_{2}, \\
D_{X} \xi_{1} & =-f_{*}\left(S_{1} X\right)+\tau_{11}(X) \xi_{1}+\tau_{12}(X) \xi_{2} \\
D_{X} \xi_{2} & =-f_{*}\left(S_{2} X\right)+\tau_{21}(X) \xi_{1}+\tau_{22}(X) \xi_{2} .
\end{aligned}
$$

Then $\nabla$ is a torsion-free affine connection on $M$, which depends only on $\mathcal{N}$ and not on the choice of local basis $\left\{\xi_{1}, \xi_{2}\right\}$. We call it the affine connection induced by $\mathcal{N}$. The other objects $h_{i}, S_{i}, \tau_{i j}, i, j \in\{1,2\}$, are respectively the affine fundamental forms, the shape operators and the normal connection forms.

\section{Para-Complex Affine Hypersurfaces}

Fist we recall some basic concepts related to para-complex geometry. For details see $[6,7]$ and [5].

A para-complex structure on a real finite dimensional vector space $V$ is an endomorphism $\widetilde{J} \in \operatorname{End}(V)$, such that $\widetilde{J}^{2}=$ id and the two eigenspaces $V^{ \pm}:=\operatorname{ker}(\mathrm{id} \mp \widetilde{J})$ of $\widetilde{J}$ have the same dimension. An almost para-complex structure on a smooth manifold $M$ is a $(1,1)$-tensor $\widetilde{J}$ on $M$ such that, for all $p \in M, \widetilde{J}_{p}$ is a para-complex structure on $T_{p} M$. An almost para-complex structure $\widetilde{J}$ on $M$ is called integrable if the distributions $D^{ \pm}:=\operatorname{ker}(\mathrm{id} \mp \widetilde{J})$ are integrable. An integrable almost para-complex structure on $M$ is called a paracomplex structure and a manifold $M$ endowed with a para-complex structure is called a para-complex manifold.

Lemma 3.1. [7] An almost para-complex structure $\widetilde{J}$ is integrable if and only if $N_{\widetilde{J}}=0$, where $N_{\widetilde{J}}$ is the Nijenhuis tensor for $\widetilde{J}$.

Let us denote by $\widetilde{\mathbb{C}}$ the real algebra of para-complex numbers, which is generated by 1 and the para-complex unit $e\left(e^{2}=1\right)$. For every $z=x+e y \in \widetilde{\mathbb{C}}$ we have the para-complex conjugation $\overline{x+e y}:=x-e y$ and the real and imaginary parts of $z: \Re(z):=x$ and $\Im(z):=y$. The free $\widetilde{\mathbb{C}}$-module $\widetilde{\mathbb{C}}^{n}$ is a para-complex vector space, where the para-complex structure is just the 
multiplication by $e$. The para-complex conjugation extends componentwise to $\widetilde{\mathbb{C}}^{n}$. The para-complex dimension of a para-complex manifold $M$ is the integer $n=\operatorname{dim}_{\widetilde{\mathbb{C}}} M:=\frac{\operatorname{dim} M}{2}$.

Let $\left(M, \widetilde{J}_{M}\right)$ and $\left(N, \widetilde{J}_{N}\right)$ be para-complex manifolds. A smooth function $f:\left(M, \widetilde{J}_{M}\right) \rightarrow\left(N, \widetilde{J}_{N}\right)$ is called para-holomorphic if $d f \circ \widetilde{J}_{M}=\widetilde{J}_{N} \circ d f$. A paraholomorphic map $f:\left(M, \widetilde{J}_{M}\right) \rightarrow \widetilde{\mathbb{C}}$ is called a para-holomorphic function.

Let $g: M^{2 n} \rightarrow \mathbb{R}^{2 n+2}$ be an immersion and let $\widetilde{J}$ be the standard paracomplex structure on $\mathbb{R}^{2 n+2}$. That is

$$
\widetilde{J}\left(x_{1}, \ldots, x_{n+1}, y_{1}, \ldots, y_{n+1}\right):=\left(y_{1}, \ldots, y_{n+1}, x_{1}, \ldots, x_{n+1}\right) .
$$

We always identify $\left(\mathbb{R}^{2 n+2}, \widetilde{J}\right)$ with $\widetilde{\mathbb{C}}^{n+1}$.

Assume now that $g_{*}(T M)$ is $\widetilde{J}$-invariant and $\left.\widetilde{J}\right|_{g_{*}\left(T_{x} M\right)}$ is a para-complex structure on $g_{*}\left(T_{x} M\right)$ for every $x \in M$. Then $\widetilde{J}$ induces an almost paracomplex structure on $M$, which we will also denote by $\widetilde{J}$. Moreover, since $\left(\mathbb{R}^{2 n+2}, \widetilde{J}\right)$ is para-complex then $(M, \widetilde{J})$ is para-complex as well. By assumption we have that $d g \circ \widetilde{J}=\widetilde{J} \circ d g$ that is $g: M^{2 n} \rightarrow \mathbb{R}^{2 n+2} \cong \widetilde{\mathbb{C}}^{n+1}$ is a paraholomorphic immersion. Since para-complex dimension of $M$ is $n$, immersion $g$ is called a para-holomorphic hypersurface.

Let $g: M^{2 n} \rightarrow \mathbb{R}^{2 n+2}$ be an affine hypersurface of codimension 2 with a transversal bundle $\mathcal{N}$. If $g$ is para-holomorphic then it is called affine paraholomorphic hypersurface. If additionally the transversal bundle $\mathcal{N}$ is $\widetilde{J}$-invariant then $g$ is called a para-complex affine hypersurface.

Let $g: M^{2 n} \rightarrow \mathbb{R}^{2 n+2}$ be a para-holomorphic hypersurface. We say that $g$ is para-complex centro-affine hypersurface if $\{g, \widetilde{J} g\}$ is a transversal bundle for $g$.

Now, let $g: M^{2 n} \rightarrow \mathbb{R}^{2 n+2}$ be a para-holomorphic hypersurface. Then for every $x \in M$ there exists a neighborhood $U$ of $x$ and a transversal vector field $\zeta: U \rightarrow \mathbb{R}^{2 n+2}$ such that $\{\zeta, \widetilde{J} \zeta\}$ is a transversal bundle for $\left.g\right|_{U}$. That is $\left.g\right|_{U}$ considered with $\{\zeta, \widetilde{J} \zeta\}$ is a para-complex affine hypersurface. Indeed, let $N_{x}$ be any vector space transversal to $g_{*}\left(T_{x} M\right)$. If $N_{x}$ is $\widetilde{J}$-invariant then it must be a para-complex vector space, so we can find vector $v \in N_{x}$ such that $\{v, \widetilde{J} v\}$ is a basis for $N_{x}$. If $N_{x}$ is not $\widetilde{J}$-invariant then $N_{x} \cap \widetilde{J} N_{x}$ must be 1-dimensional. In this case we can choose $v \in N_{x}$ such that $v \notin N_{x} \cap \widetilde{J} N_{x}$. Now vector $\widetilde{J} v$ is transversal to $g_{*}\left(T_{x} M\right)$ and linearly independent with $v$. That is $\{v, \widetilde{J} v\}$ is a para-complex transversal vector space to $g_{*}\left(T_{x} M\right)$. Summarizing at $x$ we can always find a transversal vector $v$ such that $g_{*}\left(T_{x} M\right) \oplus \operatorname{span}\{v, \widetilde{J} v\}=\mathbb{R}^{2 n+2}$. Hence, in a neighborhood of $x$ we can find a transversal vector field $\zeta$ such that $\{\zeta, \widetilde{J} \zeta\}$ is a transversal bundle for $g$ in this neighborhood.

Let $g: M^{2 n} \rightarrow \mathbb{R}^{2 n+2}$ be a para-holomorphic hypersurface and let $\zeta: U \rightarrow$ $\mathbb{R}^{2 n+2}$ be a local transversal vector field on $U \subset M$ such that $\{\zeta, \widetilde{J} \zeta\}$ is a transversal bundle to $g$. So for all tangent vector fields $X, Y \in \mathcal{X}(U)$ we can 
decompose $D_{X} Y$ and $D_{X} \zeta$ into tangent and transversal part. So we have

$\mathrm{D}_{X} g_{*} Y=g_{*}\left(\nabla_{X} Y\right)+h_{1}(X, Y) \zeta+h_{2}(X, Y) \widetilde{J} \zeta \quad$ (formula of Gauss)

$\mathrm{D}_{X} \zeta=-g_{*}(S X)+\tau_{1}(X) \zeta+\tau_{2}(X) \widetilde{J} \zeta \quad$ (formula of Weingarten)

where $\nabla$ is a torsion free affine connection on $U, h_{1}$ and $h_{2}$ are symmetric bilinear forms on $U, S$ is a $(1,1)$-tensor field on $U$ and $\tau_{1}$ and $\tau_{2}$ are 1-forms on $U$.

Using the fact that $D \widetilde{J}=0$ and the formula of Gauss by straightforward computations we can prove the following

Lemma 3.2. [5]

$$
\begin{aligned}
\nabla \widetilde{J} & =0, \\
h_{1}(X, \widetilde{J} Y) & =h_{1}(\widetilde{J} X, Y)=h_{2}(X, Y), \\
h_{2}(X, \widetilde{J} Y) & =h_{1}(X, Y) .
\end{aligned}
$$

We say that a hypersurface is nondegenerate if $h_{1}$ (and in consequence $\left.h_{2}\right)$ is nondegenerate.

Lemma 3.3. Let $g: M \rightarrow \mathbb{R}^{2 n+2}$ be a para-complex affine hypersurface with a transversal bundle $\{\zeta, \widetilde{J} \zeta\}$. Then the induced connection $\nabla$, the affine fundamental forms $h_{1}, h_{2}$, the shape operator $S$ and the transversal connection forms $\tau_{1}, \tau_{2}$ satisfy the following equations:

$$
\begin{aligned}
R(X, Y) Z= & h_{1}(Y, Z) S X+h_{2}(Y, Z) \widetilde{J}(S X) \\
& -h_{1}(X, Z) S Y-h_{2}(X, Z) \widetilde{J}(S Y), \\
\left(\nabla_{X} h_{1}\right)(Y, Z)-\left(\nabla_{Y} h_{1}\right)(X, Z)= & \tau_{1}(Y) h_{1}(X, Z)+\tau_{2}(Y) h_{2}(X, Z) \\
& -\tau_{1}(X) h_{1}(Y, Z)-\tau_{2}(X) h_{2}(Y, Z), \\
\left(\nabla_{X} h_{2}\right)(Y, Z)-\left(\nabla_{Y} h_{2}\right)(X, Z)= & \tau_{1}(Y) h_{2}(X, Z)+\tau_{2}(Y) h_{1}(X, Z) \\
& -\tau_{1}(X) h_{2}(Y, Z)-\tau_{2}(X) h_{1}(Y, Z), \\
\left(\nabla_{X} S\right)(Y)-\left(\nabla_{Y} S\right)(X)= & \tau_{1}(X) S Y+\tau_{2}(X) \widetilde{J}(S Y) \\
& -\tau_{1}(Y) S X-\tau_{2}(Y) \widetilde{J}(S X), \\
h_{1}(X, S Y)-h_{1}(S X, Y)= & 2 d \tau_{1}(X, Y), \\
h_{2}(X, S Y)-h_{2}(S X, Y)= & 2 d \tau_{2}(X, Y) .
\end{aligned}
$$

Assume now that $\{\widetilde{\zeta}, \widetilde{J} \widetilde{\zeta}\}$ is any other transversal bundle on $U$. Then there exist functions $\varphi, \psi$ on $U$ and $Z \in \mathcal{X}(U)$ such that

$$
\widetilde{\zeta}=\varphi \zeta+\psi \widetilde{J} \zeta+g_{*} Z
$$

Since $\{\widetilde{\zeta}, \widetilde{J} \widetilde{\zeta}\}$ is transversal the above formula implies that $\varphi^{2}-\psi^{2} \neq 0$. Indeed, we have

$$
\varphi \widetilde{\zeta}-\psi \widetilde{J} \widetilde{\zeta}=\left(\varphi^{2}-\psi^{2}\right) \zeta+\varphi g_{*} Z-\psi \widetilde{J} g_{*} Z
$$


If $\varphi^{2}-\psi^{2}=0$ then $\varphi \widetilde{\zeta}-\psi \widetilde{J} \widetilde{\zeta} \in T U$, but since $\{\widetilde{\zeta}, \widetilde{J} \widetilde{\zeta}\}$ is transversal we obtain $\varphi=\psi=0$, what is impossible because $\widetilde{\zeta}$ is transversal.

By the formulas of Gauss and Weingarten with respect to $\widetilde{\zeta}$ we obtain the objects $\widetilde{\nabla}, \widetilde{h_{1}}, \widetilde{h_{2}}, \widetilde{S}, \widetilde{\tau_{1}}, \widetilde{\tau_{2}}$ which satisfy the following relations

\section{Lemma 3.4.}

$$
\begin{aligned}
& h_{1}(X, Y)=\varphi \widetilde{h_{1}}(X, Y)+\psi \widetilde{h_{2}}(X, Y) \\
& h_{2}(X, Y)=\psi \widetilde{h_{1}}(X, Y)+\varphi \widetilde{h_{2}}(X, Y) \\
& \nabla_{X} Y=\widetilde{\nabla} X+\widetilde{h_{1}}(X, Y) Z+\widetilde{h_{2}}(X, Y) \widetilde{J} Z \\
& \quad-\varphi S X-\psi S X+\nabla_{X} Z=-\widetilde{S} X+\widetilde{\tau_{1}}(X) Z+\widetilde{\tau_{2}}(X) \widetilde{J} Z \\
& X(\varphi)+\varphi \tau_{1}(X)+\psi \tau_{2}(X)+h_{1}(X, Z)=\varphi \widetilde{\tau_{1}}(X)+\psi \widetilde{\tau_{2}}(X) \\
& \varphi \tau_{2}(X)+X(\psi)+\psi \tau_{1}(X)+h_{2}(X, Z)=\psi \widetilde{\tau_{1}}(X)+\varphi \widetilde{\tau_{2}}(X) \\
& \widetilde{h_{1}}=\frac{h_{1} \varphi-h_{2} \psi}{\varphi^{2}-\psi^{2}}, \\
& \widetilde{\tau_{1}}(X)=\frac{1}{2} X\left(\ln \left|\varphi^{2}-\psi^{2}\right|\right)+\tau_{1}(X)+\frac{1}{\varphi^{2}-\psi^{2}}\left(\varphi h_{1}(X, Z)-\psi h_{2}(X, Z)\right) .
\end{aligned}
$$

Proof. Formulas (3.10)-(3.15) are straightforward. Formulas (3.16) and (3.17) follow at once from (3.10), (3.11), (3.14) and (3.15).

On $U$ we define the volume form $\theta_{\zeta}$ by the formula

$$
\theta_{\zeta}\left(X_{1}, \ldots, X_{2 n}\right):=\operatorname{det}\left(g_{*} X_{1}, \ldots, g_{*} X_{2 n}, \zeta, \widetilde{J} \zeta\right)
$$

for tangent vectors $X_{i}, i=1, \ldots, 2 n$. Then, consider the function $H_{\zeta}$ on $U$ defined by

$$
H_{\zeta}:=\operatorname{det}\left[h_{1}\left(X_{i}, X_{j}\right)\right]_{i, j=1 \ldots 2 n}
$$

where $X_{1}, \ldots, X_{2 n}$ is a local basis in $T U$ such that $\theta_{\zeta}\left(X_{1}, \ldots, X_{2 n}\right)=1$. This definition is independent of the choice of basis. It is easy to see that $\nabla, \theta_{\zeta}$ and $\tau_{1}$ are related by the following formula:

$$
\nabla_{X} \theta_{\zeta}=2 \tau_{1}(X) \theta_{\zeta}
$$

If $\{\widetilde{\zeta}, \widetilde{J \zeta}\}$ is other transversal bundle on $U$ then we have the following relations between $\theta_{\widetilde{\zeta}}, H_{\widetilde{\zeta}}$ and $\theta_{\zeta}, H_{\zeta}$

\section{Lemma 3.5.}

$$
\begin{aligned}
\theta_{\widetilde{\zeta}} & =\left(\varphi^{2}-\psi^{2}\right) \theta_{\zeta}, \\
H_{\widetilde{\zeta}} & =\frac{1}{\left(\varphi^{2}-\psi^{2}\right)^{n+2}} \cdot H_{\zeta}
\end{aligned}
$$


Proof. Since Formula (3.19) is straightforward it is enough to prove (3.20). Let $\left\{X_{1}, \widetilde{J} X_{1}, \ldots, X_{n}, \widetilde{J} X_{n}\right\}$ be a local basis on $T M$. Then

$$
\theta_{\zeta}\left(X_{1}, \widetilde{J} X_{1}, \ldots, X_{n}, \widetilde{J} X_{n}\right)=\alpha
$$

where $\alpha \neq 0$ ( either $\alpha<0$ or $\alpha>0$ ). Now let $\widetilde{X_{1}}:=\frac{X_{1}}{\sqrt{|\alpha|}}$ then

$$
\theta_{\zeta}\left(\widetilde{X_{1}}, \widetilde{J} \widetilde{X_{1}}, X_{2}, \widetilde{J} X_{2}, \ldots, X_{n}, \widetilde{J} X_{n}\right)=\frac{\alpha}{|\alpha|}
$$

It follows that we can choose the basis $\left\{X_{1}, \widetilde{J} X_{1}, \ldots, X_{n}, \widetilde{J} X_{n}\right\}$ such that

$$
\theta_{\zeta}\left(X_{1}, \widetilde{J} X_{1}, \ldots, X_{n}, \widetilde{J} X_{n}\right)= \pm 1
$$

Let $Y_{i}=\frac{X_{i}}{\left|\varphi^{2}-\psi^{2}\right|^{\frac{1}{2 n}}}$ for $i=1, \ldots, n$. Then

$$
\begin{aligned}
\theta_{\widetilde{\zeta}}\left(Y_{1}, \ldots, \widetilde{J} Y_{n}\right) & =\left(\varphi^{2}-\psi^{2}\right) \theta_{\zeta}\left(Y_{1}, \ldots, \widetilde{J} Y_{n}\right) \\
& =\left(\varphi^{2}-\psi^{2}\right) \cdot \frac{1}{\left|\varphi^{2}-\psi^{2}\right|} \theta_{\zeta}\left(X_{1}, \ldots, \widetilde{J} X_{n}\right) \\
& =\operatorname{sgn}\left(\varphi^{2}-\psi^{2}\right) \theta_{\zeta}\left(X_{1}, \ldots, \widetilde{J} X_{n}\right)= \pm 1
\end{aligned}
$$

and in consequence

$$
\begin{aligned}
& H_{\widetilde{\zeta}}=\operatorname{det}\left[\begin{array}{cccc}
\widetilde{h_{1}}\left(Y_{1}, Y_{1}\right) & \widetilde{h_{1}}\left(Y_{1}, \widetilde{J} Y_{1}\right) & \cdots & \widetilde{h_{1}}\left(Y_{1}, \widetilde{J} Y_{n}\right) \\
\vdots & \vdots & \ddots & \vdots \\
\widetilde{h_{1}}\left(\widetilde{J} Y_{n}, Y_{1}\right) & \widetilde{h_{1}}\left(\widetilde{J} Y_{n}, \widetilde{J} Y_{1}\right) & \cdots & \widetilde{h_{1}}\left(\widetilde{J} Y_{n}, \widetilde{J} Y_{n}\right)
\end{array}\right] \\
&= \frac{1}{\left(\varphi^{2}-\psi^{2}\right)^{2}} \operatorname{det}\left[\begin{array}{cccc}
\widetilde{h_{1}}\left(X_{1}, X_{1}\right) & \widetilde{h_{1}}\left(X_{1}, \widetilde{J} X_{1}\right) & \cdots & \widetilde{h_{1}}\left(X_{1}, \widetilde{J} X_{n}\right) \\
\vdots & \vdots & \ddots & \vdots \\
\widetilde{h_{1}}\left(\widetilde{J} X_{n}, X_{1}\right) & \widetilde{h_{1}}\left(\widetilde{J} X_{n}, \widetilde{J} X_{1}\right) & \cdots & \widetilde{h_{1}}\left(\widetilde{J} X_{n}, \widetilde{J} X_{n}\right)
\end{array}\right] .
\end{aligned}
$$

We also have

$$
\begin{aligned}
\operatorname{det}\left[\begin{array}{cc}
\widetilde{h_{1}}\left(X_{k}, X_{l}\right) & \widetilde{h_{1}}\left(X_{k}, \widetilde{J} X_{l}\right) \\
\widetilde{h_{1}}\left(X_{m}, X_{l}\right) & \widetilde{h_{1}}\left(X_{m}, \widetilde{J} X_{l}\right)
\end{array}\right] \\
=\frac{1}{\left(\varphi^{2}-\psi^{2}\right)^{2}} \operatorname{det}\left[\begin{array}{cc}
\varphi h_{1}\left(X_{k}, X_{l}\right)-\psi h_{2}\left(X_{k}, X_{l}\right) & \varphi h_{1}\left(X_{k}, \widetilde{J} X_{l}\right)-\psi h_{2}\left(X_{k}, \widetilde{J} X_{l}\right) \\
\varphi h_{1}\left(X_{m}, X_{l}\right)-\psi h_{2}\left(X_{m}, X_{l}\right) & \varphi h_{1}\left(X_{m}, \widetilde{J} X_{l}\right)-\psi h_{2}\left(X_{m}, \widetilde{J} X_{l}\right)
\end{array}\right] \\
=\frac{1}{\left(\varphi^{2}-\psi^{2}\right)^{2}} \operatorname{det}\left[\begin{array}{cc}
\varphi h_{1}\left(X_{k}, X_{l}\right)-\psi h_{1}\left(X_{k}, \widetilde{J} X_{l}\right) & \varphi h_{1}\left(X_{k}, \widetilde{J} X_{l}\right)-\psi h_{1}\left(X_{k}, X_{l}\right) \\
\varphi h_{1}\left(X_{m}, X_{l}\right)-\psi h_{1}\left(X_{m}, \widetilde{J} X_{l}\right) & \varphi h_{1}\left(X_{m}, \widetilde{J} X_{l}\right)-\psi h_{1}\left(X_{m}, X_{l}\right)
\end{array}\right] \\
=\frac{1}{\left(\varphi^{2}-\psi^{2}\right)^{2}} \operatorname{det}\left[\begin{array}{ll}
\varphi h_{1}\left(X_{k}, X_{l}\right) & \varphi h_{1}\left(X_{k}, \widetilde{J} X_{l}\right) \\
\varphi h_{1}\left(X_{m}, X_{l}\right) & \varphi h_{1}\left(X_{m}, \widetilde{J} X_{l}\right)
\end{array}\right] \\
+\frac{1}{\left(\varphi^{2}-\psi^{2}\right)^{2}} \operatorname{det}\left[\begin{array}{ll}
-\psi h_{1}\left(X_{k}, \widetilde{J} X_{l}\right) & -\psi h_{1}\left(X_{k}, X_{l}\right) \\
-\psi h_{1}\left(X_{m}, \widetilde{J} X_{l}\right) & -\psi h_{1}\left(X_{m}, X_{l}\right)
\end{array}\right] \\
=\frac{\varphi^{2}-\psi^{2}}{\left(\varphi^{2}-\psi^{2}\right)^{2}} \operatorname{det}\left[\begin{array}{cc}
h_{1}\left(X_{k}, X_{l}\right) & h_{1}\left(X_{k}, \widetilde{J} X_{l}\right) \\
h_{1}\left(X_{m}, X_{l}\right) & h_{1}\left(X_{m}, \widetilde{J} X_{l}\right)
\end{array}\right]
\end{aligned}
$$


Now we obtain

$$
\operatorname{det}\left[\begin{array}{cc}
\widetilde{h_{1}}\left(X_{k}, X_{l}\right) & \widetilde{h_{1}}\left(X_{k}, \widetilde{J} X_{l}\right) \\
\widetilde{h_{1}}\left(X_{m}, X_{l}\right) & \widetilde{h_{1}}\left(X_{m}, \widetilde{J} X_{l}\right)
\end{array}\right]=\frac{1}{\varphi^{2}-\psi^{2}} \operatorname{det}\left[\begin{array}{cc}
h_{1}\left(X_{k}, X_{l}\right) & h_{1}\left(X_{k}, \widetilde{J} X_{l}\right) \\
h_{1}\left(X_{m}, X_{l}\right) & h_{1}\left(X_{m}, \widetilde{J} X_{l}\right)
\end{array}\right] .
$$

The above implies that

and eventually

$$
H_{\widetilde{\zeta}}=\frac{1}{\left(\varphi^{2}-\psi^{2}\right)^{2}} \cdot \frac{1}{\left(\varphi^{2}-\psi^{2}\right)^{n}} \cdot H_{\zeta}
$$

$$
H_{\widetilde{\zeta}}=\frac{1}{\left(\varphi^{2}-\psi^{2}\right)^{n+2}} \cdot H_{\zeta}
$$

When $g$ is nondegenerate there exist transversal vector fields $\zeta$ satisfying the following two conditions:

$$
\begin{aligned}
& \left|H_{\zeta}\right|=1, \\
& \tau_{1}=0 .
\end{aligned}
$$

Such vector fields are called affine normal vector fields. The first condition is a kind of normalization and the second condition implies that $\nabla \theta_{\zeta}=0$ [see (3.18) formula].

Indeed, let $\{\zeta, \widetilde{J} \zeta\}$ be an arbitrary transversal bundle for $g$. Since $g$ is nondegenerate we have $H_{\zeta} \neq 0$, so we can find functions $\varphi$ and $\psi$ such that $\varphi^{2}-\psi^{2} \neq 0$ and

$$
\left|\left(\varphi^{2}-\psi^{2}\right)^{n+2}\right|=\left|H_{\zeta}\right|
$$

Let $\widetilde{\zeta}:=\varphi \zeta+\psi \zeta+Z$ where $Z$ is an arbitrary vector field on $M$. Lemma 3.5 (Formula (3.20)] and (3.21) imply that $\left|H_{\widetilde{\zeta}}\right|=1$. We shall show that we can choose $Z$ in such a way that $\widetilde{\zeta}$ is an affine normal vector field.

By Lemma 3.4 [Formula (3.17)] we have

$$
\widetilde{\tau_{1}}(X)=\frac{1}{2} X\left(\ln \left|\varphi^{2}-\psi^{2}\right|\right)+\tau_{1}(X)+\frac{1}{\varphi^{2}-\psi^{2}}\left(\varphi h_{1}(X, Z)-\psi h_{2}(X, Z)\right)
$$

Now using Lemma 3.2 we obtain

$$
\widetilde{\tau_{1}}(X)=\frac{1}{2} X\left(\ln \left|\varphi^{2}-\psi^{2}\right|\right)+\tau_{1}(X)+\frac{1}{\varphi^{2}-\psi^{2}} \cdot h_{1}(X, \varphi Z-\psi \widetilde{J} Z) .
$$

Since $h_{1}$ is nondegenerate we can find $Z$ such that $\widetilde{\tau_{1}}(X)=0$ for all vector fields $X$ defined on $U$. In this way we have shown that on every paraholomorphic hypersurface one may find (at least locally) an affine normal vector field.

Lemma 3.6. Let $g: M^{2 n} \rightarrow \mathbb{R}^{2 n+2}$ be a nondegenerate para-holomorphic hypersurface and let $\zeta, \widetilde{\zeta}: U \rightarrow \mathbb{R}^{2 n+2}$ be two affine normal vector fields on $U \subset M$. Then $\widetilde{\zeta}=\varphi \zeta+\psi \widetilde{J} \zeta$, where $\left|\varphi^{2}-\psi^{2}\right|=1$. 
Proof. Since $\zeta, \widetilde{\zeta}$ are transversal there exist functions $\varphi, \psi \in C^{\infty}(U)$ and a tangent vector field $Z \in \mathcal{X}(U)$ such that $\widetilde{\zeta}=\varphi \zeta+\psi \widetilde{J} \zeta+Z$. Since $\left|H_{\zeta}\right|=$ $\left|H_{\widetilde{\zeta}}\right|=1$ the Formula (3.20) implies that $\left|\varphi^{2}-\psi^{2}\right|=1$. Now, due to the fact that $\tau_{1}=\widetilde{\tau}_{1}=0$ and by Formula (3.17) and Lemma 3.2 we obtain

$0=\varphi h_{1}(X, Z)-\psi h_{2}(X, Z)=\varphi h_{1}(X, Z)-\psi h_{1}(X, \widetilde{J} Z)=h_{1}(X, \varphi Z-\psi \widetilde{J} Z)$

for all $X \in \mathcal{X}(U)$. Since $h_{1}$ is nondegenerate and $\varphi^{2}-\psi^{2} \neq 0$ the last formula implies that $Z=0$. The proof is completed.

Lemma 3.7. Let $g: M \rightarrow \mathbb{R}^{2 n+2}$ be a para-complex affine hypersurface with a transversal bundle $\{\zeta, \widetilde{J} \zeta\}$. Then for each point $x \in M$ there exists a local coordinate system $x_{1}, \ldots, x_{n}, y_{1}, \ldots, y_{n}$ with origin at $x$ such that $\partial_{x_{1}}, \ldots, \partial_{x_{n}}$ and $\partial_{y_{1}}, \ldots, \partial_{y_{n}}$ are local bases for $\mathrm{D}^{+}$and $\mathrm{D}^{-}$respectively and

$$
\begin{aligned}
& h_{1}\left(\partial_{x_{i}}, \partial_{y_{j}}\right)=0, \\
& h_{2}\left(\partial_{x_{i}}, \partial_{y_{j}}\right)=0, \\
& \nabla_{\partial_{x_{i}}} \partial_{y_{j}}=\nabla_{\partial_{y_{j}}} \partial_{x_{i}}=0, \\
& \nabla_{\partial_{x_{i}}} \partial_{x_{j}} \in \mathrm{D}^{+}, \\
& \nabla_{\partial_{y_{i}}} \partial_{y_{j}} \in \mathrm{D}^{-}
\end{aligned}
$$

for $i, j=1, \ldots, n$.

Proof. Since $\mathrm{D}^{+}$and $\mathrm{D}^{-}$are involutive and $\mathrm{D}^{+} \oplus \mathrm{D}^{-}=T M$ using lemma about direct product of involutive distributions (see Prop. 5.2, p. 182 in [9]) we have that for each $x \in M$ there exists a neighbourhood $U$ of $x$ and a local coordinate system $x_{1}, \ldots, x_{n}, y_{1}, \ldots, y_{n}$ on $U$ such that $\partial_{x_{i}} \in \mathrm{D}^{+}, \partial_{y_{i}} \in \mathrm{D}^{-}$ for $i=1, \ldots, n$. Lemma 3.2 implies that

$$
h_{1}\left(\partial_{x_{i}}, \widetilde{J} \partial_{y_{j}}\right)=h_{1}\left(\widetilde{J} \partial_{x_{i}}, \partial_{y_{j}}\right) .
$$

Since $\widetilde{J} \partial_{x_{i}}=\partial_{x_{i}}$ and $\widetilde{J} \partial_{y_{j}}=-\partial_{y_{j}}$ we have $h_{1}\left(\widetilde{J} \partial_{x_{i}}, \partial_{y_{j}}\right)=h_{1}\left(\partial_{x_{i}}, \partial_{y_{j}}\right)$ that is $h_{1}\left(\partial_{x_{i}}, \partial_{y_{j}}\right)=0$ for $i, j=1, \ldots, n$. As an immediate consequence we get that $h_{2}\left(\partial_{x_{i}}, \partial_{y_{j}}\right)=0$ for $i, j=1, \ldots, n$ as well.

From (3.1) we obtain

$$
-\nabla_{\partial_{x_{i}}} \partial_{y_{j}}=\nabla_{\partial_{x_{i}}} \widetilde{J} \partial_{y_{j}}=\widetilde{J}\left(\nabla_{\partial_{x_{i}}} \partial_{y_{j}}\right)
$$

and

$$
\nabla_{\partial_{y_{j}}} \partial_{x_{i}}=\nabla_{\partial_{y_{j}}} \widetilde{J} \partial_{x_{i}}=\widetilde{J}\left(\nabla_{\partial_{y_{j}}} \partial_{x_{i}}\right),
$$

so $\nabla_{\partial_{x_{i}}} \partial_{y_{j}} \in \mathrm{D}^{-}$and $\nabla_{\partial_{y_{j}}} \partial_{x_{i}} \in \mathrm{D}^{+}$. Since $\nabla$ is torsion free we also have $\nabla_{\partial_{x_{i}}} \partial_{y_{j}}=\nabla_{\partial_{y_{j}}} \partial_{x_{i}}$ that is

$$
\nabla_{\partial_{x_{i}}} \partial_{y_{j}}=\nabla_{\partial_{y_{j}}} \partial_{x_{i}}=0
$$

Using again Formula (3.1) we get

$$
\nabla_{\partial_{x_{i}}} \partial_{x_{j}}=\nabla_{\partial_{x_{i}}} \widetilde{J} \partial_{x_{j}}=\widetilde{J}\left(\nabla_{\partial_{x_{i}}} \partial_{x_{j}}\right)
$$


and

$$
-\nabla_{\partial_{y_{i}}} \partial_{y_{j}}=\nabla_{\partial_{y_{i}}} \widetilde{J} \partial_{y_{j}}=\widetilde{J}\left(\nabla_{\partial_{y_{i}}} \partial_{y_{j}}\right)
$$

that is $\nabla_{\partial_{x_{i}}} \partial_{x_{j}} \in \mathrm{D}^{+}$and $\nabla_{\partial_{y_{i}}} \partial_{y_{j}} \in \mathrm{D}^{-}$for $i, j=1, \ldots, n$. The proof is completed.

As an immediate consequence of the above lemma we obtain

Corollary 3.1. Let $g: M \rightarrow \mathbb{R}^{2 n+2}$ be a para-complex affine hypersurface with a transversal bundle $\{\zeta, \widetilde{J} \zeta\}$. Then for each $X \in \mathrm{D}^{+}, Y \in \mathrm{D}^{-}$we have

1. $h_{i}(X, Y)=0$ for $i=1,2$;

2. Distributions $\mathrm{D}^{+}$and $\mathrm{D}^{-}$are $\nabla$ parallel. That is for every $Z \in \mathcal{X}(M)$ we have $\nabla_{Z} X \in \mathrm{D}^{+}$and $\nabla_{Z} Y \in \mathrm{D}^{-}$.

Lemma 3.8. Let $g: M \rightarrow \mathbb{R}^{2 n+2}$ be a para-complex affine hypersurface with a transversal bundle $\{\zeta, \widetilde{J} \zeta\}$. Then for each point $x \in M$ there exists a local coordinate system $x_{1}, \ldots, x_{n}, y_{1}, \ldots, y_{n}$ with origin at $x$ such that $g$ can be locally expressed in the form

$$
g\left(x_{1}, \ldots, x_{n}, y_{1}, \ldots, y_{n}\right)=A\left(x_{1}, \ldots, x_{n}\right)+B\left(y_{1}, \ldots, y_{n}\right)
$$

where

$$
A: U_{1} \ni\left(x_{1}, \ldots, x_{n}\right) \mapsto A\left(x_{1}, \ldots, x_{n}\right) \in \mathbb{R}^{2 n+2}
$$

and

$$
B: U_{2} \ni\left(y_{1}, \ldots, y_{n}\right) \mapsto B\left(y_{1}, \ldots, y_{n}\right) \in \mathbb{R}^{2 n+2}
$$

are smooth immersions from open subsets $U_{1}, U_{2} \subset \mathbb{R}^{n}$. Moreover $\widetilde{J} A=A$ and $\widetilde{J} B=-B$.

Proof. Let $x \in M$ and let $x_{1}, \ldots, x_{n}, y_{1}, \ldots, y_{n}$ be a local coordinate system from Lemma 3.7. By formula of Gauss we have

$$
g_{x_{i} y_{j}}=D_{\partial_{x_{i}}} g_{*} \partial_{y_{j}}=g_{*} \nabla_{\partial_{x_{i}}} \partial_{y_{j}}+h_{1}\left(\partial_{x_{i}}, \partial_{y_{j}}\right) \zeta+h_{2}\left(\partial_{x_{i}}, \partial_{y_{j}}\right) \tilde{J} \zeta .
$$

Now (3.22)-(3.24) imply that $g_{x_{i} y_{j}}=0$ for $i, j=1, \ldots, n$. Solving this system of partial differential equations we immediately get that there exist open subsets $U_{1}, U_{2} \subset \mathbb{R}^{n}$ and smooth functions $\bar{A}: U_{1} \rightarrow \mathbb{R}^{2 n+2}, \bar{B}: U_{2} \rightarrow \mathbb{R}^{2 n+2}$ such that

$$
g\left(x_{1}, \ldots, x_{n}, y_{1}, \ldots, y_{n}\right)=\bar{A}\left(x_{1}, \ldots, x_{n}\right)+\bar{B}\left(y_{1}, \ldots, y_{n}\right)
$$

for $\left(x_{1}, \ldots, x_{n}\right) \in U_{1}$ and $\left(y_{1}, \ldots, y_{n}\right) \in U_{2}$. Since $g$ is an immersion it is obvious that both $\bar{A}$ and $\bar{B}$ are immersions too. To prove the last part of the lemma it is enough to note that since $g$ is para-holomorphic we have $\bar{A}_{x_{i}}=g_{*}\left(\partial_{x_{i}}\right)=\widetilde{J} g_{*}\left(\partial_{x_{i}}\right)=\widetilde{J} \bar{A}_{x_{i}}$ and $-\bar{B}_{y_{i}}=-g_{*}\left(\partial_{y_{i}}\right)=\widetilde{J} g_{*}\left(\partial_{y_{i}}\right)=\widetilde{J} \bar{B}_{y_{i}}$ for $i=1, \ldots, n$. That is there exist constants $C_{1}, C_{2} \in \mathbb{R}^{2 n+2}$ such that $\widetilde{J} \bar{A}=$ $\bar{A}+C_{1}$ and $\widetilde{J} \bar{B}=-\bar{B}+C_{2}$. Note that $\widetilde{J} C_{1}=-C_{1}$ and $\widetilde{J} C_{2}=C_{2}$. Let us define 
$A:=\bar{A}+\frac{1}{2} C_{1}+\frac{1}{2} C_{2}$ and $B:=\bar{B}-\frac{1}{2} C_{1}-\frac{1}{2} C_{2}$. Then we have $A+B=\bar{A}+\bar{B}=g$ and

$$
\begin{aligned}
\widetilde{J} A=\widetilde{J} \bar{A}-\frac{1}{2} C_{1}+\frac{1}{2} C_{2} & =\bar{A}+C_{1}-\frac{1}{2} C_{1}+\frac{1}{2} C_{2} \\
& =\bar{A}+\frac{1}{2} C_{1}+\frac{1}{2} C_{2}=A \\
\widetilde{J} B=\widetilde{J} \bar{B}+\frac{1}{2} C_{1}-\frac{1}{2} C_{2} & =-\bar{B}+C_{2}+\frac{1}{2} C_{1}-\frac{1}{2} C_{2} \\
& =-\bar{B}+\frac{1}{2} C_{1}+\frac{1}{2} C_{2}=-B .
\end{aligned}
$$

\section{Para-Complex Affine Hyperspheres}

In this section we focus on a special type of para-complex hypersurfaces. Namely, we study so called para-complex affine hyperspheres. The definition of para-complex affine hypersphere is very similar to definition of a hypersphere in a complex case. The aim of this section is to give a complete local classification of such hypersurfaces. Especially, we shall show that there is a strict correspondence between real and para-complex affine hyperspheres.

A nondegenerate para-complex hypersurface is said to be a proper paracomplex affine hypersphere if there exists an affine normal vector field $\zeta$ such that $S=\alpha I$, where $\alpha \in \mathbb{R} \backslash\{0\}$ and $\tau_{2}=0$. If there exists an affine normal vector field $\zeta$ such that $S=0$ and $\tau_{2}=0$ we say about an improper paracomplex affine hypersphere.

Remark 4.1. Let $g: M \rightarrow \mathbb{R}^{2 n+2}$ be a proper para-complex affine hypersphere with a transversal bundle $\{\zeta, \widetilde{J} \zeta\}$ such that $S=\alpha I$ for $\zeta$. Then $g$ is a paracomplex affine hypersphere with a transversal bundle $\{\widetilde{\zeta}, \widetilde{J} \widetilde{\zeta}\}$, where $\widetilde{\zeta}=\frac{1}{2}(\alpha+$ $\left.\frac{1}{\alpha}\right) \zeta+\frac{1}{2}\left(\frac{1}{\alpha}-\alpha\right) \widetilde{J} \zeta$ and $\widetilde{S}=$ id.

Now we shall prove a classification theorem for para-complex affine hyperspheres.

Theorem 4.1. Let $g: M \rightarrow \mathbb{R}^{2 n+2}$ be a para-complex affine hypersphere with a transversal bundle $\{\zeta, \widetilde{J} \zeta\}$. Then there exist open subsets $U_{1} \subset \mathbb{R}^{n}, U_{2} \subset \mathbb{R}^{n}$ and (real) affine hyperspheres

$$
f_{1}: U_{1} \rightarrow \mathbb{R}^{n+1}, \quad f_{2}: U_{2} \rightarrow \mathbb{R}^{n+1}
$$

such that $g$ can be locally expressed in the form

$$
g=f_{1} \times f_{2}+\widetilde{J} \circ\left(f_{1} \times\left(-f_{2}\right)\right) .
$$

Moreover, if $g$ is proper (respectively improper) then both $f_{1}$ and $f_{2}$ are proper (respectively improper) as well. The converse is also true, in the sense, that for every two proper (respectively improper) affine hyperspheres $f_{1}$ and $f_{2}$ the 
Formula (4.1) defines a proper (respectively improper) para-complex affine hypersphere.

Proof. Let $g: M \rightarrow \mathbb{R}^{2 n+2}$ be a para-complex affine hypersphere and let $x \in$ $M$. Since $g$ is a para-complex affine hypersurface the Lemma 3.8 implies that there exist open subsets $U_{1}, U_{2} \subset \mathbb{R}^{n}$ and smooth immersions $A: U_{1} \rightarrow \mathbb{R}^{2 n+2}$, $B: U_{2} \rightarrow \mathbb{R}^{2 n+2}$ such that $\widetilde{J} A=A, \widetilde{J} B=-B$ and $g$ can be expressed in some neighborhood of $x$ in the form:

$$
g: U_{1} \times U_{2} \ni\left(x_{1}, \ldots, x_{n}, y_{1}, \ldots, y_{n}\right) \mapsto A\left(x_{1}, \ldots, x_{n}\right)+B\left(y_{1}, \ldots, y_{n}\right) \in \mathbb{R}^{2 n+2} .
$$

Let $\nabla, h_{1}, S, \tau_{1}$ and $\tau_{2}$ be induced affine objects for $g$. Since $g$ is a hypersphere we have $\tau_{1}=\tau_{2}=0$ and $S=\alpha$ id for some $\alpha \in \mathbb{R}$.

Let $\pi_{1}: \mathbb{R}^{2 n+2} \rightarrow \mathbb{R}^{n+1}$ be a projection of first $(n+1)$ variables on $\mathbb{R}^{n+1}$ and let $\pi_{2}: \mathbb{R}^{2 n+2} \rightarrow \mathbb{R}^{n+1}$ be a projection of last $(n+1)$ variables on $\mathbb{R}^{n+1}$. Let us define $f_{1}: U_{1} \ni\left(x_{1}, \ldots, x_{n}\right) \mapsto \pi_{1} \circ A\left(x_{1}, \ldots, x_{n}\right) \in \mathbb{R}^{n+1}$ and $f_{2}: U_{2} \ni$ $\left(y_{1}, \ldots, y_{n}\right) \mapsto \pi_{2} \circ B\left(y_{1}, \ldots, y_{n}\right) \in \mathbb{R}^{n+1}$. Since $A$ and $B$ are immersions and $\widetilde{J} A=A$ and $\widetilde{J} B=-B$ we easily verify that $f_{1}$ and $f_{2}$ are immersions too. We also have

$$
g=f_{1} \times f_{2}+\widetilde{J} \circ\left(f_{1} \times\left(-f_{2}\right)\right) .
$$

Now, it is enough to show that $f_{1}$ and $f_{2}$ are affine hyperspheres. For this purpose we shall consider the following two cases.

Case I $\alpha \neq 0$. In this case we have $\zeta=-\alpha g$. Since $\zeta$ and $\widetilde{J} \zeta$ are linearly independent and transversal to $g$ then also $\frac{1}{2}(\zeta+\widetilde{J} \zeta)=-\alpha A$ and $\frac{1}{2}(\zeta-\widetilde{J} \zeta)=-\alpha B$ are transversal to $g$. In particular $\left\{A_{x_{1}}, \ldots, A_{x_{n}}, A\right\}$ and $\left\{B_{y_{1}}, \ldots, B_{y_{n}}, B\right\}$ are linearly independent. Let $\alpha_{1}, \ldots, \alpha_{n}, \beta$ be functions on $U_{1}$ such that

$$
\sum_{i} \alpha_{i} f_{1_{x_{i}}}+\beta f_{1}=0
$$

Then

$$
\begin{aligned}
\sum_{i} \alpha_{i} A_{x_{i}}+\beta A & =\sum_{i} \alpha_{i}\left(\pi_{1} A_{x_{i}}, \pi_{1} A_{x_{i}}\right)+\beta\left(\pi_{1} A, \pi_{1} A\right) \\
& =\left(\sum_{i} \alpha_{i} f_{1_{x_{i}}}+\beta f_{1}, \sum_{i} \alpha_{i} f_{1_{x_{i}}}+\beta f_{1}\right)=(0,0) .
\end{aligned}
$$

Since $\left\{A_{x_{1}}, \ldots, A_{x_{n}}, A\right\}$ are linearly independent the above implies that $\alpha_{1}=$ $\cdots=\alpha_{n}=\beta=0$ that is $f_{1}$ is linearly independent with $\left\{f_{1_{x_{i}}}\right\}_{i=1}^{n}$. Now $\xi_{1}:=-2 \alpha f_{1}$ is a transversal vector field to $f_{1}$. In a similar way we show that $\xi_{2}:=-2 \alpha f_{2}$ is a transversal vector field to $f_{2}$.

The Gauss formula for $g$ implies that

$$
\begin{aligned}
D_{\partial_{x_{i}}} g_{*} \partial_{x_{j}} & =g_{*}\left(\nabla_{\partial_{x_{i}}} \partial_{x_{j}}\right)+h_{1}\left(\partial_{x_{i}}, \partial_{x_{j}}\right) \zeta+h_{2}\left(\partial_{x_{i}}, \partial_{x_{j}}\right) \widetilde{J} \zeta \\
& =\Gamma_{i j}^{k} g_{x_{k}}+h_{1}\left(\partial_{x_{i}}, \partial_{x_{j}}\right)(\zeta+\widetilde{J} \zeta) \\
& =\Gamma_{i j}^{k} A_{x_{k}}+h_{1}\left(\partial_{x_{i}}, \partial_{x_{j}}\right) \cdot(-2 \alpha A),
\end{aligned}
$$


where $\Gamma_{i j}^{k}$ are Christoffel's symbols for $\nabla$ and we used the fact that $h_{1}=h_{2}$ on $\mathrm{D}^{+}$. On the other hand we have

$$
D_{\partial_{x_{i}}} g_{*} \partial_{x_{j}}=g_{x_{i} x_{j}}=A_{x_{i} x_{j}}=\left(f_{1_{x_{i} x_{j}}}, f_{1_{x_{i} x_{j}}}\right) .
$$

Using (4.3) in (4.2) and applying $\pi_{1}$ projection we get

$$
\begin{aligned}
f_{1_{x_{i} x_{j}}} & =\Gamma_{i j}^{k} f_{1_{x_{k}}}+h_{1}\left(\partial_{x_{i}}, \partial_{x_{j}}\right) \cdot\left(-2 \alpha f_{1}\right) \\
& =\Gamma_{i j}^{k} f_{1_{x_{k}}}+h_{1}\left(\partial_{x_{i}}, \partial_{x_{j}}\right) \xi_{1} .
\end{aligned}
$$

For $f_{1}$ we have the Gauss formula, that is

$$
f_{1_{x_{i} x_{j}}}=D_{\partial_{x_{i}}} f_{1_{*}} \partial_{x_{j}}=f_{1 *}\left(\nabla_{\partial_{x_{i}}}^{+} \partial_{x_{j}}\right)+h^{+}\left(\partial_{x_{i}}, \partial_{x_{j}}\right) \xi_{1},
$$

where $\nabla^{+}$is the induced connection and $h^{+}$is the second fundamental form for $f_{1}$. Now (4.4) implies that $\nabla^{+}=\left.\nabla\right|_{T U_{1} \times T U_{1}}$ and $h^{+}=\left.h_{1}\right|_{T U_{1} \times T U_{1}}$. In particular $h^{+}$is nondegenerate since $h_{1}$ is nondegenerate on $T U_{1} \times T U_{1}$. Note also that for $f_{1}$ we have the induced volume element $\theta^{+}$given by the formula

$$
\begin{aligned}
\theta^{+}\left(\partial_{x_{1}}, \ldots, \partial_{x_{n}}\right): & =\operatorname{det}\left[f_{1_{x_{1}}}, \ldots, f_{1_{x_{n}}}, \xi_{1}\right] \\
& =-2 \alpha \operatorname{det}\left[f_{1_{x_{1}}}, \ldots, f_{1_{x_{n}}}, f_{1}\right] .
\end{aligned}
$$

In a similar way like above (but now using the fact that $h_{2}=-h_{1}$ on $\left.\mathrm{D}^{-}\right)$we obtain that $\nabla^{-}=\left.\nabla\right|_{T U_{2} \times T U_{2}}, h^{-}=\left.h_{1}\right|_{T U_{2} \times T U_{2}}$ and

$$
\theta^{-}\left(\partial_{y_{1}}, \ldots, \partial_{y_{n}}\right)=-2 \alpha \operatorname{det}\left[f_{2 y_{1}}, \ldots, f_{2 y_{n}}, f_{2}\right] \text {, }
$$

where $\nabla^{-}$is the induced connection, $h^{-}$is the second fundamental form and $\theta^{-}$is the induced volume element for $f_{2}$.

Let $\theta$ be the induced volume element for $g$, that is

$$
\begin{aligned}
\theta\left(\partial_{x_{1}}, \ldots, \partial_{x_{n}}, \partial_{y_{1}}, \ldots, \partial_{y_{n}}\right)= & \operatorname{det}\left[A_{x_{1}}, \ldots, A_{x_{n}}, B_{y_{1}}, \ldots, B_{y_{n}}\right. \\
& -\alpha(A+B),-\alpha(A-B)] \\
= & \alpha^{2} \operatorname{det}\left[A_{x_{1}}, \ldots, A_{x_{n}}, B_{y_{1}}, \ldots, B_{y_{n}}, A,-B\right] \\
& +\alpha^{2} \operatorname{det}\left[A_{x_{1}}, \ldots, A_{x_{n}}, B_{y_{1}}, \ldots, B_{y_{n}}, B, A\right] \\
= & -2 \alpha^{2} \operatorname{det}\left[A_{x_{1}}, \ldots, A_{x_{n}}, B_{y_{1}}, \ldots, B_{y_{n}}, A, B\right] \\
= & -2 \alpha^{2} \cdot(-1)^{n} \operatorname{det}\left[A_{x_{1}}, \ldots, A_{x_{n}}, A, B_{y_{1}}, \ldots, B_{y_{n}}, B\right] .
\end{aligned}
$$

Let us denote

$$
\begin{aligned}
M & :=\left[A_{x_{1}}, \ldots, A_{x_{n}}, A, B_{y_{1}}, \ldots, B_{y_{n}}, B\right], \\
M^{+} & :=\left[\pi_{1} A_{x_{1}}, \ldots, \pi_{1} A_{x_{n}}, \pi_{1} A\right], \\
M^{-} & :=\left[\pi_{2} B_{y_{1}}, \ldots, \pi_{2} B_{y_{n}}, \pi_{2} B\right] .
\end{aligned}
$$

Then $M$ can be expressed in the following block form:

$$
M=\left[\begin{array}{cc}
M^{+} & -M^{-} \\
M^{+} & M^{-}
\end{array}\right] .
$$


Now replacing the row $i$ with the sum of rows $i$ and $i+n+1$ for $i=1, \ldots, n+1$, we obtain a new matrix

$$
M^{\prime}=\left[\begin{array}{cc}
2 M^{+} & 0 \\
M^{+} & M^{-}
\end{array}\right]
$$

It is easy to see that

$$
\begin{aligned}
\operatorname{det} M & =\operatorname{det} M^{\prime}=\operatorname{det}\left(2 M^{+}\right) \cdot \operatorname{det}\left(M^{-}\right) \\
& =2^{n+1} \operatorname{det} M^{+} \cdot \operatorname{det} M^{-} \\
& =\frac{2^{n-1}}{\alpha^{2}} \theta^{+}\left(\partial_{x_{1}}, \ldots, \partial_{x_{n}}\right) \cdot \theta^{-}\left(\partial_{y_{1}}, \ldots, \partial_{y_{n}}\right) .
\end{aligned}
$$

Finally we get the following relation between $\theta, \theta^{+}$and $\theta^{-}$:

$$
\theta\left(\partial_{x_{1}}, \ldots, \partial_{x_{n}}, \partial_{y_{1}}, \ldots, \partial_{y_{n}}\right)=2^{n} \cdot(-1)^{n+1} \theta^{+}\left(\partial_{x_{1}}, \ldots, \partial_{x_{n}}\right) \theta^{-}\left(\partial_{y_{1}}, \ldots, \partial_{y_{n}}\right) .
$$

Let det $h_{1}$ be the determinant of $h_{1}$ in the basis $\left\{\partial_{x_{1}}, \ldots, \partial_{x_{n}}, \partial_{y_{1}}, \ldots, \partial_{y_{n}}\right\}$. Since $h_{1}\left(\partial_{x_{i}}, \partial_{y_{j}}\right)=0$ for $i, j=1, \ldots, n$ we have that

$$
\operatorname{det} h_{1}=\operatorname{det} h^{+} \cdot \operatorname{det} h^{-},
$$

where $\operatorname{det} h^{+}$is the determinant of $h^{+}$with respect to the basis $\left\{\partial_{x_{1}}, \ldots, \partial_{x_{n}}\right\}$ and $\operatorname{det} h^{-}$is the determinant of $h^{-}$with respect to the basis $\left\{\partial_{y_{1}}, \ldots, \partial_{y_{n}}\right\}$. Now using (4.5), (4.6) and the fact that $\left|H_{\zeta}\right|=1$ we obtain

$$
\begin{aligned}
1=\left|H_{\zeta}\right| & =\left|\frac{\operatorname{det} h_{1}}{\theta^{2}}\right|=\left|\frac{\operatorname{det} h^{+} \cdot \operatorname{det} h^{-}}{2^{2 n}\left(\theta^{+}\right)^{2} \cdot\left(\theta^{-}\right)^{2}}\right| \\
& =\frac{1}{2^{2 n}}\left(\frac{\omega_{h^{+}}\left(\partial_{x_{1}}, \ldots, \partial_{x_{n}}\right)}{\theta^{+}\left(\partial_{x_{1}}, \ldots, \partial_{x_{n}}\right)}\right)^{2} \cdot\left(\frac{\omega_{h^{-}}\left(\partial_{y_{1}}, \ldots, \partial_{y_{n}}\right)}{\theta^{-}\left(\partial_{y_{1}}, \ldots, \partial_{y_{n}}\right)}\right)^{2} .
\end{aligned}
$$

That is

$$
\left|\frac{\omega_{h+}\left(\partial_{x_{1}}, \ldots, \partial_{x_{n}}\right)}{\theta^{+}\left(\partial_{x_{1}}, \ldots, \partial_{x_{n}}\right)}\right| \cdot\left|\frac{\omega_{h^{-}}\left(\partial_{y_{1}}, \ldots, \partial_{y_{n}}\right)}{\theta^{-}\left(\partial_{y_{1}}, \ldots, \partial_{y_{n}}\right)}\right|=2^{n} .
$$

Since $\omega_{h^{+}}, \theta^{+}$depends only on $x_{1}, \ldots, x_{n}$ and $\omega_{h^{-}}, \theta^{-}$depends only on $y_{1}, \ldots, y_{n}$ the last equality implies that both $\omega_{h^{+}} / \theta^{+}$and $\omega_{h^{-}} / \theta^{-}$are constant. So there exist constants $c^{+}$and $c^{-}$such that $\omega_{h^{+}}=c^{+} \theta^{+}$and $\omega_{h^{-}}=c^{-} \theta^{-}$.

Case II $\alpha=0$. Without loss of generality we may assume that $\zeta=(0, \ldots, 0,1)$ $\in \mathbb{R}^{2 n+2}$. Let us denote $\xi_{1}=\xi_{2}=(0, \ldots, 0,1) \in \mathbb{R}^{n+1}$. Since $\{\zeta, \widetilde{J} \zeta\}$ is transversal to $g$ we have that $\zeta+\widetilde{J} \zeta=\left(\xi_{1}, \xi_{1}\right)$ is transversal to $g$ as well. Let $\alpha_{1}, \ldots, \alpha_{n}, \beta$ be functions on $U_{1}$ such that

$$
\sum_{i} \alpha_{i} f_{1_{i}}+\beta \xi_{1}=0
$$


Then

$$
\begin{aligned}
& \sum_{i} \alpha_{i} A_{x_{i}}+\beta(\zeta+\widetilde{J} \zeta) \\
& \quad=\sum_{i} \alpha_{i}\left(\pi_{1} A_{x_{i}}, \pi_{1} A_{x_{i}}\right)+\beta\left(\xi_{1}, \xi_{1}\right) \\
& \quad=\left(\sum_{i} \alpha_{i} f_{1_{x_{i}}}+\beta \xi_{1}, \sum_{i} \alpha_{i} f_{1_{x_{i}}}+\beta \xi_{1}\right)=(0,0) .
\end{aligned}
$$

Now, since $\left\{g_{x_{1}}, \ldots, g_{x_{n}}, \zeta+\widetilde{J} \zeta\right\}$ are linearly independent it immediately follows that $\alpha_{1}=\cdots=\alpha_{n}=\beta=0$ and in consequence $\xi_{1}$ is transversal to $f_{1}$. In a similar way we show that $\xi_{2}$ is transversal to $f_{2}$. Like for $\alpha \neq 0$, using the Gauss formulas for $g, f_{1}$ and $f_{2}$, we obtain that $h^{+}=h_{1}$ on $\mathrm{D}^{+}, h^{-}=h_{1}$ on $\mathrm{D}^{-}$and $\operatorname{det} h_{1}=\operatorname{det} h^{+} \cdot \operatorname{det} h^{-}$. In particular we get that both $f_{1}$ and $f_{2}$ are nondegenerate.

For the induced volume $\theta$ we have

$$
\begin{aligned}
& \theta\left(\partial_{x_{1}}, \ldots, \partial_{x_{n}}, \partial_{y_{1}}, \ldots, \partial_{y_{n}}\right) \\
& \quad=\operatorname{det}\left[A_{x_{1}}, \ldots, A_{x_{n}}, B_{y_{1}}, \ldots, B_{y_{n}}, \zeta, \widetilde{J} \zeta\right] \\
& \quad=\frac{1}{2} \cdot(-1)^{n+1} \operatorname{det}\left[A_{x_{1}}, \ldots, A_{x_{n}}, \zeta+\widetilde{J} \zeta, B_{y_{1}}, \ldots, B_{y_{n}}, \zeta-\widetilde{J} \zeta\right] .
\end{aligned}
$$

The above implies that

$\theta\left(\partial_{x_{1}}, \ldots, \partial_{x_{n}}, \partial_{y_{1}}, \ldots, \partial_{y_{n}}\right)=2^{n} \cdot(-1)^{n+1} \theta^{+}\left(\partial_{x_{1}}, \ldots, \partial_{x_{n}}\right) \cdot \theta^{-}\left(\partial_{y_{1}}, \ldots, \partial_{y_{n}}\right)$, where $\theta^{+}$and $\theta^{-}$are the induced volume forms for $f_{1}$ and $f_{2}$ respectively. Now, since $\zeta$ is affine normal, we have

$$
\begin{aligned}
1=\left|H_{\zeta}\right| & =\left|\frac{\operatorname{det} h_{1}}{(\theta)^{2}}\right|=\left|\frac{\operatorname{det} h^{+} \cdot \operatorname{det} h^{-}}{4^{n}\left(\theta^{+} \cdot \theta^{-}\right)^{2}}\right| \\
& =\frac{1}{4^{n}}\left|\frac{\omega_{h^{+}}}{\theta^{+}}\right|^{2} \cdot\left|\frac{\omega_{h^{-}}}{\theta^{-}}\right|^{2} \cdot
\end{aligned}
$$

Since $\omega_{h^{+}}, \theta^{+}$depends only on $x_{1}, \ldots, x_{n}$ and $\omega_{h^{-}}, \theta^{-}$depends only on $y_{1}, \ldots, y_{n}$ the last equality implies that both $\omega_{h^{+}} / \theta^{+}$and $\omega_{h^{-}} / \theta^{-}$are constant and in consequence $f_{1}$ and $f_{2}$ are improper affine hyperspheres.

In order to prove the converse assume that $f_{1}: U_{1} \rightarrow \mathbb{R}^{n+1}$ and $f_{2}: U_{2} \rightarrow$ $\mathbb{R}^{n+1}$ are two affine hyperspheres with the Blaschke field $\xi_{1}$ and $\xi_{2}$ respectively. Let us denote $U=U_{1} \times U_{2}$ and let $g: U \rightarrow \mathbb{R}^{2 n+2}$ be defined by the Formula (4.1) that is

$$
\begin{aligned}
g\left(x_{1}, \ldots, x_{n}, y_{1}, \ldots, y_{n}\right)= & \left(f_{1}\left(x_{1}, \ldots, x_{n}\right), f_{1}\left(x_{1}, \ldots, x_{n}\right)\right) \\
& +\left(-f_{2}\left(y_{1}, \ldots, y_{n}\right), f_{2}\left(y_{1}, \ldots, y_{n}\right)\right) .
\end{aligned}
$$

For the above and similar expressions we will often ommit arguments using the following short notation:

$$
g=\left(f_{1}, f_{1}\right)+\left(-f_{2}, f_{2}\right)
$$


Like in the proof of the first part of the theorem we shall consider two cases.

Case $\mathbf{I} f_{1}$ and $f_{2}$ are proper affine hyperspheres. In this case we have $\xi_{1}=$ $-\lambda_{1} f_{1}$ and $\xi_{2}=-\lambda_{2} f_{2}$ for some $\lambda_{1}, \lambda_{2} \in \mathbb{R}_{+}$. Let us define $\zeta:=-\alpha g$, where

$$
\alpha:=\left(\frac{1}{2}\right)^{\frac{2 n+2}{n+2}} \cdot \sqrt{\lambda_{1} \lambda_{2}} .
$$

We shall show that $g$ with $\zeta$ is a para-complex affine hypersphere. For this purpose let $\alpha_{1}, \ldots, \alpha_{n}, \beta_{1}, \ldots, \beta_{n}, \gamma, \delta \in C^{\infty}(U)$ and

$$
\sum \alpha_{i} g_{x_{i}}+\sum \beta_{i} g_{y_{i}}+\gamma \zeta+\delta \widetilde{J} \zeta=0 .
$$

Since $g_{x_{i}}=\left(f_{1_{x_{i}}}, f_{1_{x_{i}}}\right)$ and $g_{y_{i}}=\left(-f_{2 y_{i}}, f_{2 y_{i}}\right)$ we obtain

$$
\sum\left(\alpha_{i} f_{1_{x_{i}}}-\beta_{i} f_{2 y_{i}}\right)-\alpha(\gamma+\delta) f_{1}-\alpha(\delta-\gamma) f_{2}=0
$$

and

$$
\sum\left(\alpha_{i} f_{1_{x_{i}}}+\beta_{i} f_{2 y_{i}}\right)-\alpha(\gamma+\delta) f_{1}-\alpha(\gamma-\delta) f_{2}=0 .
$$

The above implies that

$$
\sum \alpha_{i} f_{x_{i}}-\alpha(\gamma+\delta) f_{1}=0
$$

and

$$
\sum \beta_{i} f_{2 y_{i}}-\alpha(\gamma-\delta) f_{2}=0 .
$$

Since $f_{1}$ and $f_{2}$ are proper affine hyperspheres then $\left\{f_{1_{x_{1}}}, \ldots, f_{1_{x_{n}}}, f_{1}\right\}$ as well as $\left\{f_{2 y_{1}}, \ldots, f_{2 y_{n}}, f_{2}\right\}$ are linearly independent, that is

$$
\alpha_{1}=\cdots=\alpha_{n}=0, \gamma+\delta=0
$$

and

$$
\beta_{1}=\cdots=\beta_{n}=0, \gamma-\delta=0 .
$$

In particular $\gamma=\delta=0$. In this way we have shown that

$$
\left\{g_{x_{1}}, \ldots, g_{x_{n}}, g_{y_{1}}, \ldots, g_{y_{n}}, \zeta, \widetilde{J} \zeta\right\}
$$

are linearly independent. Since $\widetilde{J} g_{x_{i}}=g_{x_{i}}$ and $\widetilde{J} g_{y_{i}}=-g_{y_{i}}$ we see that $g$ is a para-complex hypersurface with a transversal bundle $\{\zeta, \widetilde{J} \zeta\}$. The Weingarten formula for $g$ immediately implies that $S=\alpha$ id and $\tau_{1}=\tau_{2}=0$, so it is enough to show that $g$ is nondegenerate and $\left|H_{\zeta}\right|=1$. For this purpose note that since $\partial_{x_{i}} \in \mathrm{D}^{+}$and $\partial_{y_{i}} \in \mathrm{D}^{-}$we have $h_{1}\left(\partial_{x_{i}} \partial_{x_{j}}\right)=h_{2}\left(\partial_{x_{i}}, \partial_{x_{j}}\right), h_{1}\left(\partial_{y_{i}} \partial_{y_{j}}\right)=$ $-h_{2}\left(\partial_{y_{i}}, \partial_{y_{j}}\right)$ and $h_{1}\left(\partial_{x_{i}}, \partial_{y_{j}}\right)=h_{2}\left(\partial_{x_{i}}, \partial_{y_{j}}\right)=0$ for $i, j=1, \ldots, n$. Now using the Gauss formula we get

$$
g_{x_{i} x_{j}}=g_{*}\left(\nabla_{\partial_{x_{i}}} \partial_{x_{j}}\right)-2 \alpha h_{1}\left(\partial_{x_{i}}, \partial_{x_{j}}\right)\left(f_{1}, f_{1}\right) .
$$

On the other hand

$$
g_{x_{i} x_{j}}=\left(f_{1_{x_{i} x_{j}}}, f_{1_{x_{i} x_{j}}}\right)=\left(f_{1_{*}}\left(\nabla_{\partial_{x_{i}}}^{+} \partial_{x_{j}}\right), f_{1_{*}}\left(\nabla_{\partial_{x_{i}}}^{+} \partial_{x_{j}}\right)\right)
$$




$$
-h^{+}\left(\partial_{x_{i}}, \partial_{x_{j}}\right) \lambda_{1}\left(f_{1}, f_{1}\right)
$$

where $\nabla^{+}$and $h^{+}$are the induced affine connection and the second fundamental form for $f_{1}$. Now it easily follows that

$$
h_{1}\left(\partial_{x_{i}}, \partial_{x_{j}}\right)=\frac{\lambda_{1}}{2 \alpha} h^{+}\left(\partial_{x_{i}}, \partial_{x_{j}}\right) .
$$

Similarly we obtain

$$
h_{2}\left(\partial_{y_{i}}, \partial_{y_{j}}\right)=\frac{\lambda_{2}}{2 \alpha} h^{-}\left(\partial_{y_{i}}, \partial_{y_{j}}\right),
$$

where $h^{-}$is the second fundamental form for $f_{2}$. Formulas (4.8) and (4.9) imply that

$$
\operatorname{det} h_{1}=\left(\frac{\lambda_{1}}{2 \alpha}\right)^{n} \cdot\left(\frac{\lambda_{2}}{2 \alpha}\right)^{n} \operatorname{det} h^{+} \cdot \operatorname{det} h^{-} .
$$

In particular $g$ is nondegenerate. Now we shall calculate $\theta_{\zeta}$. Namely, we have

$$
\begin{aligned}
\theta_{\zeta}\left(\partial_{x_{1}}, \ldots, \partial_{x_{n}}, \partial_{y_{1}}, \ldots, \partial_{y_{n}}\right) & =\operatorname{det}\left[g_{x_{1}}, \ldots, g_{x_{n}}, g_{y_{1}}, \ldots, g_{y_{n}}, \zeta, \widetilde{J} \zeta\right] \\
& =\alpha^{2} \operatorname{det}\left[g_{x_{1}}, \ldots, g_{x_{n}}, g_{y_{1}}, \ldots, g_{y_{n}}, g, \widetilde{J} g\right] \\
& =2 \alpha^{2} \operatorname{det}\left[g_{x_{1}}, \ldots, g_{x_{n}}, g_{y_{1}}, \ldots, g_{y_{n}}, \frac{g+\widetilde{J} g}{2}, \frac{\widetilde{J} g-g}{2}\right] \\
& =-2(-1)^{n} \alpha^{2} \operatorname{det}\left[g_{x_{1}}, \ldots, g_{x_{n}}, \frac{g+\widetilde{J} g}{2}, g_{y_{1}}, \ldots, g_{y_{n}}, \frac{g-\widetilde{J} g}{2}\right] .
\end{aligned}
$$

Let us denote

$$
M:=\left[g_{x_{1}}, \ldots, g_{x_{n}}, \frac{g+\widetilde{J} g}{2}, g_{y_{1}}, \ldots, g_{y_{n}}, \frac{g-\widetilde{J} g}{2}\right] .
$$

It is easy to see that $M$ has the following block form:

$$
M=\left[\begin{array}{cc}
M^{+} & -M^{-} \\
M^{+} & M^{-}
\end{array}\right],
$$

where

$$
M^{+}=\left[f_{1_{x_{1}}}, \ldots, f_{1_{x_{n}}}, f_{1}\right]=\left[f_{1_{x_{1}}}, \ldots, f_{1_{x_{n}}},-\frac{1}{\lambda_{1}} \cdot \xi_{1}\right]
$$

and

$$
M^{-}=\left[f_{2 y_{1}}, \ldots, f_{2 y_{n}}, f_{2}\right]=\left[f_{2 y_{1}}, \ldots, f_{2 y_{n}},-\frac{1}{\lambda_{2}} \cdot \xi_{2}\right] .
$$

Like in the first part of the proof we see that

$$
\begin{aligned}
\operatorname{det} M & =2^{n+1} \operatorname{det} M^{+} \cdot \operatorname{det} M^{-} \\
& =2^{n+1} \cdot \frac{-1}{\lambda_{1}} \cdot \theta^{+}\left(\partial_{x_{1}}, \ldots, \partial_{x_{n}}\right) \cdot \frac{-1}{\lambda_{2}} \cdot \theta^{-}\left(\partial_{y_{1}}, \ldots, \partial_{y_{n}}\right) \\
& =\frac{2^{n+1}}{\lambda_{1} \lambda_{2}} \theta^{+}\left(\partial_{x_{1}}, \ldots, \partial_{x_{n}}\right) \cdot \theta^{-}\left(\partial_{y_{1}}, \ldots, \partial_{y_{n}}\right),
\end{aligned}
$$


where $\theta^{+}$and $\theta^{-}$are the induced volume elements for $f_{1}$ and $f_{2}$ respectively. To simplify notation in the forthcoming formulas we will be omitting arguments of $\theta_{\zeta}, \theta^{+}$and $\theta^{-}$. Now we obtain

$$
\theta_{\zeta}=(-1)^{n+1} \alpha^{2} \cdot \frac{2^{n+2}}{\lambda_{1} \lambda_{2}} \theta^{+} \cdot \theta^{-} .
$$

Since $\xi_{1}$ and $\xi_{2}$ are the Blaschke fields we have $\omega_{h^{+}}=\theta^{+}$and $\omega_{h^{-}}=\theta^{-}$. In particular $\left(\theta^{+}\right)^{2}=\left|\operatorname{det} h^{+}\right|$and $\left(\theta^{-}\right)^{2}=\left|\operatorname{det} h^{-}\right|$. Now using (4.10) we obtain

$$
\begin{aligned}
\left(\theta_{\zeta}\right)^{2} & =\alpha^{4} \cdot \frac{2^{2 n+4}}{\left(\lambda_{1} \lambda_{2}\right)^{2}} \cdot\left(\theta^{+}\right)^{2} \cdot\left(\theta^{-}\right)^{2} \\
& =\alpha^{4} \cdot \frac{2^{2 n+4}}{\left(\lambda_{1} \lambda_{2}\right)^{2}} \cdot\left|\operatorname{det} h^{+}\right| \cdot\left|\operatorname{det} h^{-}\right| \\
& =\alpha^{4} \cdot \frac{2^{2 n+4}}{\left(\lambda_{1} \lambda_{2}\right)^{2}} \cdot\left(\frac{2 \alpha}{\lambda_{1}}\right)^{n} \cdot\left(\frac{2 \alpha}{\lambda_{2}}\right)^{n} \cdot\left|\operatorname{det} h_{1}\right| \\
& =\alpha^{2 n+4} \cdot \frac{2^{4 n+4}}{\left(\lambda_{1} \lambda_{2}\right)^{n+2}} \cdot\left|\operatorname{det} h_{1}\right| \\
& =\left|\operatorname{det} h_{1}\right|
\end{aligned}
$$

where the last equality is an immediate consequence of (4.7). Summarizing we have shown that

$$
\left|H_{\zeta}\right|=\left|\frac{\operatorname{det} h_{1}}{\left(\theta_{\zeta}\right)^{2}}\right|=1,
$$

that is $g$ is a proper para-complex affine hypersphere.

Case II $f_{1}$ and $f_{2}$ are improper affine hyperspheres. In this case we have $\xi_{1}=\xi_{2}=(0, \ldots, 1) \in \mathbb{R}^{n+1}$. Let us define $\zeta:=2^{\frac{-n}{n+2}}(0, \ldots, 0,1) \in \mathbb{R}^{2 n+2}$ and let $\alpha_{1}, \ldots, \alpha_{n}, \beta_{1}, \ldots, \beta_{n}, \gamma, \delta \in C^{\infty}(U)$ and

$$
\sum \alpha_{i} g_{x_{i}}+\sum \beta_{i} g_{y_{i}}+\gamma \zeta+\delta \widetilde{J} \zeta=0
$$

Like for proper hyperspheres we easily compute that

$$
\sum\left(\alpha_{i} f_{1 x_{i}}-\beta_{i} f_{2 y_{i}}\right)+2^{\frac{-n}{n+2}} \delta \xi_{1}=0
$$

and

$$
\sum\left(\alpha_{i} f_{1 x_{i}}+\beta_{i} f_{2 y_{i}}\right)+2^{\frac{-n}{n+2}} \gamma \xi_{2}=0 .
$$

The above implies that $\alpha_{i}=0, \beta_{i}=0, \gamma=\delta=0$ and in consequence

$$
\left\{g_{x_{1}}, \ldots, g_{x_{n}}, g_{y_{1}}, \ldots, g_{y_{n}}, \zeta, \widetilde{J} \zeta\right\}
$$

are linearly independent. It means that $g$ is a para-complex affine hypersurface with a transversal bundle $\{\zeta, \widetilde{J} \zeta\}$. Using similar methods like in the proof for the first case we obtain

$$
\operatorname{det} h_{1}=2^{\frac{2 n^{2}}{n+2}} \operatorname{det} h^{+} \cdot \operatorname{det} h^{-}
$$


and

$$
\theta_{\zeta}=(-1)^{n+1} 2^{n} \cdot 2^{\frac{-2 n}{n+2}} \theta^{+} \cdot \theta^{-}
$$

where $h^{+}, h^{-}$and $\theta^{+}, \theta^{-}$are the second fundamental forms and the induced volume elements for $f_{1}$ and $f_{2}$ respectively. It easily follows from (4.11) that $g$ is nondegenerate. From the Weingarten formula we have $S=0, \tau_{1}=0$ and $\tau_{2}=0$. Now (4.11) and (4.12) implies that

$$
\left|H_{\zeta}\right|=\left|\frac{2^{\frac{2 n^{2}}{n+2}} \operatorname{det} h^{+} \cdot \operatorname{det} h^{-}}{\left[(-1)^{n+1} 2^{n} \cdot 2^{\frac{-2 n}{n+2}} \cdot \theta^{+} \cdot \theta^{-}\right]^{2}}\right|=1,
$$

that is $g$ is an improper para-complex affine hypersphere. The proof is concluded.

The above theorem gives us a one-to-one correspondence between paracomplex affine hyperspheres and pairs of (real) affine hyperspheres. Now, we shall show some examples

Example 4.1. Let $g: \mathbb{R}^{2} \rightarrow \mathbb{R}^{4}$ be given by the formula

$$
g(x, y):=\lambda_{1}{ }^{-\frac{3}{4}}\left(\begin{array}{c}
\cos x \\
\sin x \\
\cos x \\
\sin x
\end{array}\right)+\lambda_{2}{ }^{-\frac{3}{4}}\left(\begin{array}{c}
-\cos y \\
-\sin y \\
\cos y \\
\sin y
\end{array}\right)
$$

where $\lambda_{1}, \lambda_{2}>0$. It easily follows that $g$ is an immersion. Moreover $\widetilde{J} g_{x}=g_{x}$ and $\widetilde{J} g_{y}=-g_{y}$, so $g$ is a para-holomorphic hypersurface. If we take $\zeta:=$ $-\left(\frac{1}{2}\right)^{\frac{4}{3}} \sqrt{\lambda_{1} \lambda_{2}} \cdot g$ then $\{\zeta, \widetilde{J} \zeta\}$ is a transversal bundle for $g$. By straightforward computations we obtain

$$
\begin{aligned}
& h_{1}=\left[\begin{array}{cc}
\frac{2^{\frac{1}{3}}}{\sqrt{\lambda_{1} \lambda_{2}}} & 0 \\
0 & \frac{2^{\frac{1}{3}}}{\sqrt{\lambda_{1} \lambda_{2}}}
\end{array}\right], \quad h_{2}=\left[\begin{array}{cc}
\frac{2^{\frac{1}{3}}}{\sqrt{\lambda_{1} \lambda_{2}}} & 0 \\
0 & -\frac{2^{\frac{1}{3}}}{\sqrt{\lambda_{1} \lambda_{2}}}
\end{array}\right], \\
& S=\left(\frac{1}{2}\right)^{\frac{4}{3}} \sqrt{\lambda_{1} \lambda_{2}} \mathrm{id}, \quad \tau_{1}=\tau_{2}=0
\end{aligned}
$$

relative to the canonical basis $\left\{\partial_{x}, \partial_{y}\right\}$. Moreover, since

$$
\theta_{\zeta}\left(\partial_{x}, \partial_{y}\right):=\operatorname{det}\left[g_{x}, g_{y}, \zeta, \widetilde{J} \zeta\right]=\frac{2^{\frac{1}{3}}}{\sqrt{\lambda_{1} \lambda_{2}}}
$$

one may easily compute that $H_{\zeta}=1$, that is $g$ is a proper para-complex affine sphere. 
Example 4.2. Let $g: \mathbb{R}^{2} \rightarrow \mathbb{R}^{4}$ be given by the formula

$$
g(x, y):=\lambda_{1}{ }^{-\frac{3}{4}}\left(\begin{array}{c}
\cosh x \\
\sinh x \\
\cosh x \\
\sinh x
\end{array}\right)+\lambda_{2}{ }^{-\frac{3}{4}}\left(\begin{array}{c}
-\cosh y \\
-\sinh y \\
\cosh y \\
\sinh y
\end{array}\right)
$$

where $\lambda_{1}, \lambda_{2}>0$. Exactly like in the previous example we have that $g$ is an immersion and $\widetilde{J} g_{x}=g_{x}$ and $\widetilde{J} g_{y}=-g_{y}$, so $g$ is a para-holomorphic hypersurface. Again taking $\zeta:=-\left(\frac{1}{2}\right)^{\frac{4}{3}} \sqrt{\lambda_{1} \lambda_{2}} \cdot g$ we obtain that $\{\zeta, \widetilde{J} \zeta\}$ is a transversal bundle for $g$. We also have

$$
\begin{aligned}
h_{1} & =\left[\begin{array}{cc}
-\frac{2^{\frac{1}{3}}}{\sqrt{\lambda_{1} \lambda_{2}}} & 0 \\
0 & -\frac{2^{\frac{1}{3}}}{\sqrt{\lambda_{1} \lambda_{2}}}
\end{array}\right], \quad h_{2}=\left[\begin{array}{cc}
-\frac{2^{\frac{1}{3}}}{\sqrt{\lambda_{1} \lambda_{2}}} & 0 \\
0 & \frac{2^{\frac{1}{3}}}{\sqrt{\lambda_{1} \lambda_{2}}}
\end{array}\right], \\
S & =\left(\frac{1}{2}\right)^{\frac{4}{3}} \sqrt{\lambda_{1} \lambda_{2}} \text { id }, \quad \tau_{1}=\tau_{2}=0
\end{aligned}
$$

relative to the canonical basis $\left\{\partial_{x}, \partial_{y}\right\}$. Moreover, since

$$
\theta_{\zeta}\left(\partial_{x}, \partial_{y}\right):=\operatorname{det}\left[g_{x}, g_{y}, \zeta, \widetilde{J} \zeta\right]=\frac{2^{\frac{1}{3}}}{\sqrt{\lambda_{1} \lambda_{2}}}
$$

we easily compute that $H_{\zeta}=1$, that is $g$ is a proper para-complex affine sphere.

Example 4.3. In this example we consider two very similar surfaces. Let $g: \mathbb{R}^{2} \rightarrow \mathbb{R}^{4}$ and $g^{\prime}: \mathbb{R}^{2} \rightarrow \mathbb{R}^{4}$ be given by the formulas:

$$
g(x, y):=\lambda_{1}-\frac{3}{4}\left(\begin{array}{c}
\cosh x \\
\sinh x \\
\cosh x \\
\sinh x
\end{array}\right)+\lambda_{2}{ }^{-\frac{3}{4}}\left(\begin{array}{c}
-\cos y \\
-\sin y \\
\cos y \\
\sin y
\end{array}\right)
$$

and

$$
g^{\prime}(x, y):=\lambda_{1}{ }^{-\frac{3}{4}}\left(\begin{array}{c}
\cos x \\
\sin x \\
\cos x \\
\sin x
\end{array}\right)+\lambda_{2}{ }^{-\frac{3}{4}}\left(\begin{array}{c}
-\cosh y \\
-\sinh y \\
\cosh y \\
\sinh y
\end{array}\right),
$$

where $\lambda_{1}, \lambda_{2}>0$. Exactly like in the previous examples we prove that $g$ and $g^{\prime}$ are para-holomorphic hypersurfaces. Let $\zeta:=-\left(\frac{1}{2}\right)^{\frac{4}{3}} \sqrt{\lambda_{1} \lambda_{2}} \cdot g$ and $\zeta^{\prime}:=$ $-\left(\frac{1}{2}\right)^{\frac{4}{3}} \sqrt{\lambda_{1} \lambda_{2}} \cdot g^{\prime}$ then $\{\zeta, \widetilde{J} \zeta\}$ and $\left\{\zeta^{\prime}, \widetilde{J} \zeta^{\prime}\right\}$ are transversal bundles for $g$ and $g^{\prime}$ respectively. For $g$ we have

$$
h_{1}=\left[\begin{array}{cc}
-\frac{2^{\frac{1}{3}}}{\sqrt{\lambda_{1} \lambda_{2}}} & 0 \\
0 & \frac{2^{\frac{1}{3}}}{\sqrt{\lambda_{1} \lambda_{2}}}
\end{array}\right], \quad h_{2}=\left[\begin{array}{cc}
-\frac{2^{\frac{1}{3}}}{\sqrt{\lambda_{1} \lambda_{2}}} & 0 \\
0 & -\frac{2^{\frac{1}{3}}}{\sqrt{\lambda_{1} \lambda_{2}}}
\end{array}\right],
$$




$$
S=\left(\frac{1}{2}\right)^{\frac{4}{3}} \sqrt{\lambda_{1} \lambda_{2}} \text { id }, \quad \tau_{1}=\tau_{2}=0
$$

and

$$
\theta_{\zeta}\left(\partial_{x}, \partial_{y}\right):=\operatorname{det}\left[g_{x}, g_{y}, \zeta, \widetilde{J} \zeta\right]=\frac{2^{\frac{1}{3}}}{\sqrt{\lambda_{1} \lambda_{2}}}
$$

relative to the canonical basis $\left\{\partial_{x}, \partial_{y}\right\}$. Now it easily follows that $H_{\zeta}=-1$ that is $g$ is a proper para-complex affine sphere. In a similar way we show that also $g^{\prime}$ is a para-complex affine sphere.

Example 4.4. Let $g: \mathbb{R}^{2} \rightarrow \mathbb{R}^{4}$ be given by the formula

$$
g(x, y):=\left(\begin{array}{c}
x \\
\frac{1}{2} x^{2} \\
x \\
\frac{1}{2} x^{2}
\end{array}\right)+\left(\begin{array}{c}
-y \\
-\frac{1}{2} y^{2} \\
y \\
\frac{1}{2} y^{2}
\end{array}\right) .
$$

It easily follows that $g$ is an immersion and $\widetilde{J} g_{x}=g_{x}$ and $\widetilde{J} g_{y}=-g_{y}$, so $g$ is a para-holomorphic hypersurface. Let $\zeta:=2^{-\frac{1}{3}}(0,0,0,1)^{T}$ then $\widetilde{J} \zeta=$ $2^{-\frac{1}{3}}(0,1,0,0)^{T}$ and $\{\zeta, \widetilde{J} \zeta\}$ is a transversal bundle for $g$. We compute

$$
h_{1}=\left[\begin{array}{cc}
2^{\frac{1}{3}} & 0 \\
0 & 2^{\frac{1}{3}}
\end{array}\right], \quad h_{2}=\left[\begin{array}{cc}
2^{\frac{1}{3}} & 0 \\
0 & -2^{\frac{1}{3}}
\end{array}\right], \quad S=0, \quad \tau_{1}=\tau_{2}=0
$$

relative to the canonical basis $\left\{\partial_{x}, \partial_{y}\right\}$. Since

$$
\theta_{\zeta}\left(\partial_{x}, \partial_{y}\right):=\operatorname{det}\left[g_{x}, g_{y}, \zeta, \widetilde{J} \zeta\right]=2^{\frac{1}{3}}
$$

then $H_{\zeta}=1$, that is $g$ is an improper para-complex affine sphere.

Using Theorem 4.1 we give a complete local classification of 1-dimensional (in para-complex sense) para-complex affine spheres. Namely we have the following theorem:

Theorem 4.2. Let $g: M^{2} \rightarrow \mathbb{R}^{4}$ be a para-complex affine hypersphere. If $g$ is proper then it can be locally expressed in one of the forms (4.13)-(4.16). If $g$ is improper then it can be locally expressed in the form (4.17).

Proof. It is well known [8] that the only (up to equiaffine transformation) 1-dimensional (real) affine spheres are a circle $\gamma_{1}(t)=k^{-\frac{3}{4}}(\cos t, \sin t)$, hyperbola $\gamma_{2}(t)=k^{-\frac{3}{4}}(\cosh t, \sinh t)$ and a parabola $\gamma_{3}(t)=\left(t, \frac{1}{2} t^{2}\right) \cdot \gamma_{1}$ and $\gamma_{2}$ are proper spheres and $\gamma_{3}$ is an improper sphere. Now, applying Theorem 4.1 we easily obtain that there are only four (up to a para-complex equiaffine transformation) proper 1-dimensional para-complex affine spheres, that is spheres from Examples 4.1, 4.2 and 4.3. Similarly the only improper 1-dimensional para-complex affine sphere is the sphere form Example 4.4.

Remark 4.2. Surfaces (4.13)-(4.17) are examples of so called translation surfaces (see $[10,11]$ for details). 
Open Access. This article is distributed under the terms of the Creative Commons Attribution 4.0 International License (http://creativecommons.org/licenses/by/4.0/), which permits unrestricted use, distribution, and reproduction in any medium, provided you give appropriate credit to the original author(s) and the source, provide a link to the Creative Commons license, and indicate if changes were made.

\section{References}

[1] Dillen, F., Vrancken, L., Verstraelen, L.: Complex affine differential geometry Atti Acc. Peloritana dei Pericolanti LXVI, pp. 232-260 (1988)

[2] Al-Aqeel, A., Bejancu, A.: On the geometry of paracomplex submanifolds. Demonstr. Math. 34(4), 919-932 (2001)

[3] Cruceanu, V., Fortuny, P., Gadea, P.M.: A survey on para-complex geometry. Rocky Mt. J. Math. 26(1), 83-115 (1996)

[4] Schäfer, L.: Para-tt*-bundles on the tangent bundle of an almost para-complex manifold. Ann. Glob. Anal. Geom. 32(2), 125-145 (2007)

[5] Lawn, M.A., Schäfer, L.: Decompositions of para-complex vector bundles and para-complex affine immersions. Results Math. 48, 246-274 (2005)

[6] Cortés, V., Lawn, M.A., Schäfer, L.: Affine hyperspheres associated to special para-Kähler manifolds. Int. J. Geom. Methods Mod. Phys. 3, 995-1009 (2006)

[7] Cortés, V., Mayer, C., Mohaupt, T., Saueressing, F.: Special geometry of Euclidean supersymmetry I: vector multiplets. J. High Energy Phys. 73, 3-28 (2004)

[8] Nomizu, K., Sasaki, T.: Affine Differential Geometry. Cambridge University Press, Cambridge (1994)

[9] Kobayashi, S., Nomizu, K.: Foundations of Differential Geometry, vol. 1. Wiley, New York (1963)

[10] Magid, M., Vrancken, L.: Affine translation surfaces with constant sectional curvature. J. Geom. 68, 192-199 (2000)

[11] Magid, M., Vrancken, L.: Affine translation surfaces. Results Math. 35, 134-144 (1999)

\section{Zuzanna Szancer}

Department of Applied Mathematics

University of Agriculture in Krakow

253 Balicka St.

30-198 Kraków

Poland

e-mail: Zuzanna.Szancer@ur.Krakow.pl

Received: September 17, 2016.

Accepted: March 22, 2017. 